\title{
État des lieux des bananeraies (Musa sp.) en zone de culture du cocotier, sur le littoral en Côte d'Ivoire : cas de la station Marc DELORME et des villages aux alentours
}

\author{
Janvier Pierre-Marie COFFI ${ }^{1,2^{*}}$, Thierry Tacra LEKADOU ${ }^{2}$, Joséphine Tamia AMA ${ }^{1}$, \\ Siaka TRAORÉ ${ }^{2}$, Didier Martial Saraka YAO ${ }^{3}$, Fernand Charly $\mathrm{AGOH}^{4,2}$, \\ Zadjehi Eric Blanchard KOFFI ${ }^{3}$, Engueran Konan DJAHA ${ }^{1}$ et François N'Klo HALA ${ }^{1}$ \\ ${ }^{1}$ Laboratoire de Géosciences et Environnement, Unité de Formation et de Recherche (UFR) des Sciences et \\ Gestion de l'Environnement, Université Nangui Abrogoua (UNA), Abidjan, Côte d'Ivoire. \\ ${ }^{2}$ Centre National de Recherche Agronomique, Station de Recherche Marc DELORME, 07 BP 13 Abidjan \\ 07, Côte d'Ivoire. \\ ${ }^{3}$ Laboratoire de Biologie et d'Amélioration des Productions Végétales, UFR Sciences de la Nature, \\ Université Péléforo Gon Coulibaly, Korhogo, Côte d'Ivoire. \\ ${ }^{4}$ Laboratoire des Sciences du Sol, de l'Eau et des Géomatériaux (LSSEG), Université Felix Houphouët \\ Boigny, Abidjan, Côte d'Ivoire. \\ *Auteur correspondant ; E-mail : janvier77@yahoo.fr, Tel : (00225) 0758076230
}

\author{
Received: 12-07-2021 \\ Accepted: 14-10-2021 \\ Published: 31-12 2021
}

\section{RÉSUMÉ}

Cette étude avait pour objectif de mieux connaître la problématique liée à la production du plantain à la Station Marc DELORME et les localités aux alentours. Pour ce faire, une enquête impliquant 130 répondants a été effectuée. Les résultats ont révélé que la production de plantain sur le littoral est plus réalisée par des hommes $(63,8)$. La tranche d'âge la plus active en ce qui concerne cette culture est celle de plus de 50 ans $(45,5 \%)$. La variété la plus rencontrée dans les plantations était la variété CORNE (74,3\%). La culture du plantain est faite en association $(66,2 \%)$ ou en monoculture $(33,8 \%)$. Au total, $62,3 \%$ des producteurs n'appliquent pas d'engrais, $77,7 \%$ ne pratiquent pas d'irrigation et $88,5 \%$ n'utilisent pas de pesticide au cours de la culture. Les bananes plantains récoltées sont plus destinées à la consommation domestique $(53,8 \%)$ qu'à la vente. En période d'abondance, les coûts fluctuent entre $500 \mathrm{~F}$ à 3000 F CFA le régime. Par contre, En période de pénurie, les coûts des régimes varient entre 1000 et 4000 F CFA. La culture du plantain peut être envisagée en association avec le cocotier vu son importance au sein des ménages.

(C) 2021 International Formulae Group. All rights reserved.

Mots clés : Production, plantain, littoral, Côte d'Ivoire. 


\title{
Status of banana plantations (Musa sp.) in coconut cultivation areas on the coast of Côte d'Ivoire: case of the Marc DELORME station and surrounding villages
}

\begin{abstract}
The aim of this study is to better understand the problems related to plantain production at the Marc DELORME Station and the surrounding localities. To this end, a survey involving 130 respondents was conducted. The results revealed that plantain production is carried out more by men $(63.8 \%)$. The most active age group with regard to this crop is the over $50 \mathrm{~s}(45.5 \%)$. The variety most frequently found in the plantations was the CORNE variety (74.3\%). Plantain is grown in association $(66.2 \%)$ or as a monoculture $(33.8 \%)$. In total, $62.3 \%$ of producers do not apply fertilizers, $77.7 \%$ do not use irrigation and $88.5 \%$ do not use pesticides during cultivation. The plantains harvested are more destined for domestic consumption (53.8\%) than for sale. In periods of abundance, costs fluctuate between $500 \mathrm{~F}$ and $3000 \mathrm{~F} \mathrm{CFA}$ per bunch. On the other hand, in periods of shortage, the costs of the bunch vary between 1000 and 4000 F CFA. The cultivation of plantain can be considered in association with the coconut tree given its importance in households.
\end{abstract}

(C) 2021 International Formulae Group. All rights reserved.

Keywords: Production, plantain, coast, Côte d'Ivoire.

\section{INTRODUCTION}

La banane est une ressource alimentaire pour 400 millions de personnes dans le monde (Loeillet, 2007). Elle est une source d'énergie essentielle pour ces populations, grâce à sa teneur élevée en glucides et son apport énergétique qui est de l'ordre de120 Kcal pour $100 \mathrm{~g}$ de banane cuite (Sharrock et Lusty, 2000). Dans les régions tropicales et subtropicales, le plantain est une denrée de base où il intervient dans la confection de plusieurs mets (Kwa et Temple, 2019) et représente une importante source de revenue pour les ménages (Dhed'a et al., 2011). En termes de production mondiale, le plantain vient en quatrième place après le riz, le blé et le maïs (Lassois et al., 2009).

En Côte d'Ivoire, le plantain est le troisième vivrier le plus cultivé après l'igname et le manioc (Traoré et al., 2009). Avec une production annuelle de 1,42 million de tonne (Kouame, 2014), les bananes plantains interviennent dans la confection de plats locaux tels que le foufou, le foutou, le cracro, l'alloko etc.

Malgré l'importance du plantain dans les ménages, sa culture reste circonscrite aux zones Est, Centre-Ouest, Sud-Ouest et Ouest (Thiémélé, 2017). Au Sud-Est littoral, l'attrait pour la culture de la banane plantain est faible devant celui du cocotier. En effet dans cette zone, la nuciculture est la principale source de revenue des populations (Assa, 2007). Cependant, elle est soumise à une rude compétition d'autres oléagineux tels que le palmier à huile, le soja, l'arachide) sur le marché international (Koffi, 2016). Couplé à ces phénomènes, les périodes d'entrée en production des cocotiers restent encore longues, malgré les avancées de la génétique ( 3 à 4 ans pour les hybrides précoces et améliorés codés PB113+ et PB121+) et, les pressions foncières sur le littoral mettent en mal la filière nucicole.

C'est dans ce cadre que la recherche s'oriente vers l'association de vivriers dans les interlignes de cocotiers afin d'améliorer le revenu des nuciculteurs. Ainsi, en prélude à une étude sur une association cocotier-bananier sur le littoral ivoirien, une enquête diagnostique s'avère nécessaire en vue de faire un état des lieux de la culture de la banane plantain. L'objectif de l'étude est de contribuer à une meilleure connaissance de la problématique liée à la production du plantain à la station Marc DELORME ainsi que les localités aux alentour, au Sud-Est d'Abidjan, en zone de culture de cocotier. 


\section{MATÉRIEL ET MÉTHODES}

\section{Site d'étude}

L'enquête s'est tenue de novembre 2019 à janvier 2020, à la station Marc DELORME et dans les localités aux alentours dans un rayon de $10 \mathrm{~km}$. La Station Marc DELORME est située dans la commune de Port-Bouët, dans le district d'Abidjan, au Sud de la Côte d'Ivoire, entre les longitudes $05^{\circ} 14^{\prime}$ et $05^{\circ} 15^{\prime} \mathrm{N}$ et les latitudes $03^{\circ} 54^{\prime}$ et $03^{\circ} 55^{\prime} \mathrm{W}$ (Figure 1). Le climat qui prévaut dans cette zone est de type Attiéen avec quatre saisons, dont deux saisons sèches et deux saisons pluvieuses. En ce qui concerne les saisons sèches, la plus longue part de décembre à mars et la courte d'août à septembre. Pour les saisons pluvieuses, la plus longue couvre la période d'avril à juillet et la courte s'étend de d'octobre à novembre. Sur la décennie allant de 2010 à 2019, la pluviométrie moyenne a été de $1811,84 \mathrm{~mm}$, les températures moyennes annuelles ont varié de 26,01 à $26,94{ }^{\circ} \mathrm{C}$. L'ensoleillement a été de l'ordre de $2000 \mathrm{~h}$.an${ }^{1}$ et l'humidité relative d'environ 80 à $90 \%$ (Station Météorologique Marc DELORME, 2020). La zone couverte est bordée par une branche de la lagune Ebrié et l'eau souterraine peut être facilement captée dans une nappe de type aquifère à $4 \mathrm{~m}$ de profondeur. Le sol est constitué de sable tertiaire typique des savanes littorales (Brou, 2005 ; Lekadou et al., 2009) et le relief est particulièrement formé de plaines basses souvent marécageuses (Diabagaté, 2008). Les sables tertiaires du littoral en Côte d'Ivoire sont composés de $0,52 \%$ de carbone, $0,05 \%$ d'azote, $0,25 \mathrm{cmol}(+) \mathrm{kg}^{-1}$ et 42,2 mg.kg- ${ }^{-1}$ de phosphore assimilable. $\mathrm{Le}^{\mathrm{pH}} \mathrm{H}_{\text {eau }} \mathrm{du}$ sol est de 5,79 (Brou, 2005).

\section{Échantillonnage et analyse des données}

La Station MARC DELORME et les villages aux alentours ont été choisis à cause de leur proximité des parcelles de cocotier. La méthodologie de recensement a été exhaustive et l'échantillonnage a été dirigé par un choix raisonné. Deux critères ont été retenus pour définir l'échantillon de producteurs :

- Etre agriculteur et vivre dans les villages à l'intérieur ou aux alentours de la Station ;

- Avoir un champ de bananier de plantain d'au moins 05 ans, contenant au moins 10 bananiers vu que la zone littorale ne fait pas partie des grandes zones de production de la banane plantain en Côte d'ivoire.

Les données ont ainsi pu être collectées sur 130 paysans. La collecte a été faite à partir d'une observation directe, consistant à effectuer un entretien individuel formel inspiré du questionnaire d'enquête de Bioversity International/CIALCA et de Bakouétila et al., (2016). Les rubriques adoptées renseignent sur :

- Le genre et le niveau de culture des paysans ;

- Les pratiques culturales mises en œuvre par les producteurs ;

- L'usage de fertilisants, de pesticides ou d'irrigation en culture ;

- La destination des bananes récoltées dans la zone et leurs coûts en période d'abondance (d'octobre à mars) et de pénurie (d'avril à septembre) (FIRCA, 2014).

Afin de permettre aux producteurs de quantifier les apports qu'ils effectuent en fiente, des dosettes pouvant contenir $5 \mathrm{~kg}$ de fiente et des balances électroniques ont été transportées sur le terrain au cours de l'enquête. Les données collectées sur les fiches ont permis d'établir une base de données, ensuite une vérification de la conformité des fiches sur la base de données collectées a été faite afin de réduire le risque d'erreur lors des saisies. L'analyse des données a été effectuée grâce au module Excel de Microsoft Office 2013, aux logiciels Sphinx Plus V5 et R version 4.0.3. 

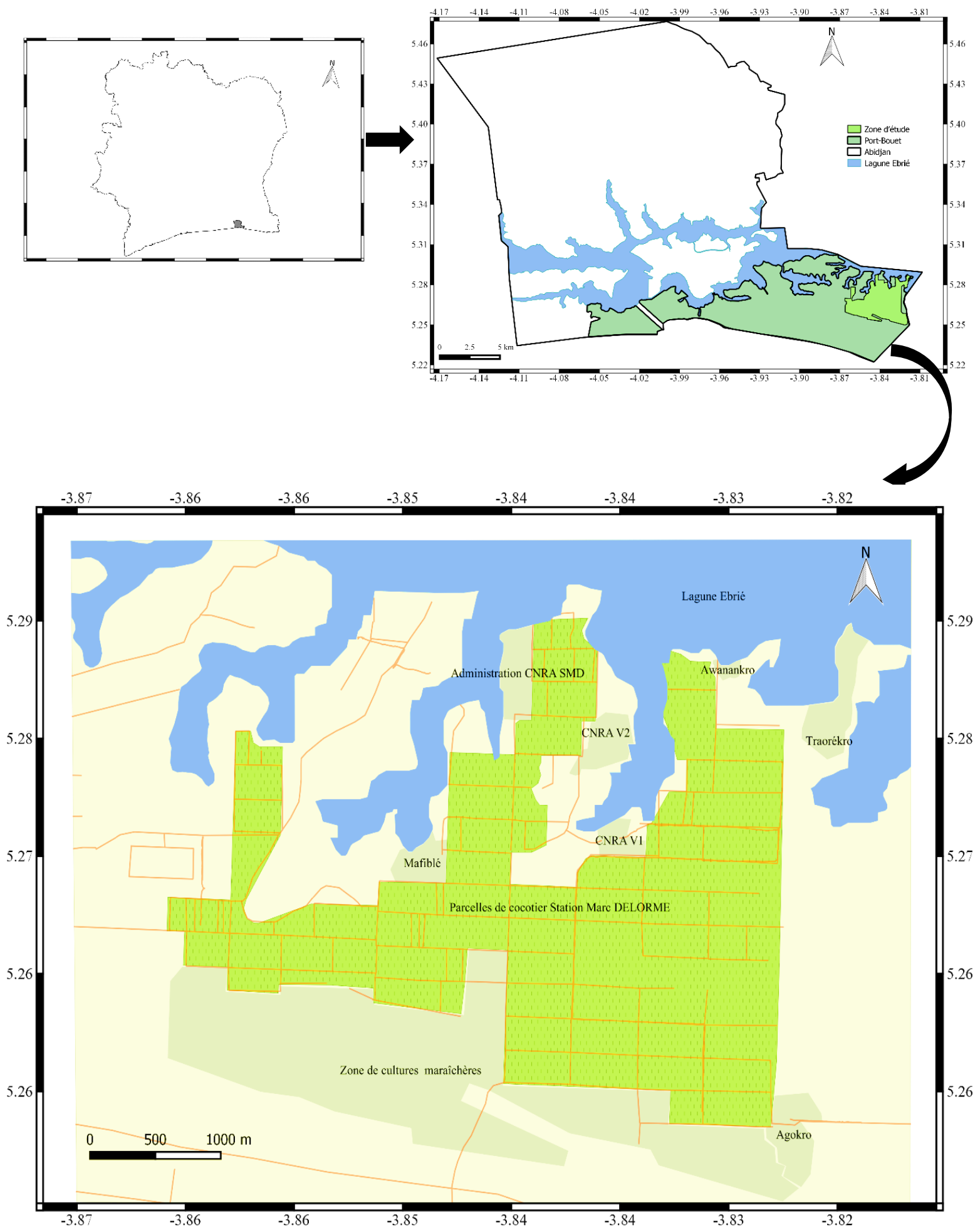

Figure 1 : Situation géographique de la station Marc DELORME et des localités environnantes. 


\section{RÉSULTATS}

\section{Caractéristiques sociodémographiques des producteurs de banane plantain}

Les investigations auprès des producteurs ont permis de révéler une forte implication des hommes $(63,8 \%)$ par rapport femmes $(36,2 \%)$ dans la culture du plantain (Tableau 1). L'enquête a révélé que $45,4 \%$ des producteurs de plantain avaient 50 ans ou plus, alors que seulement $2,3 \%$ avaient des âges inférieurs ou égaux à 20 ans (Tableau 2). La moitié des producteurs $50 \%$ a été scolarisée. Par ailleurs $23,1 \%$ ont eu un niveau secondaire, suivi de ceux ayant fait l'école primaire $(20,8 \%)$. Les producteurs ayant fait l'école coranique représentent $3,8 \%$ de l'effectif. Le plus faible taux observé (2,3\%), a été celui des producteurs ayant eu le niveau d'étude supérieur (Tableau 3). Ces producteurs étaient composés de $55,4 \%$ d'ivoiriens et les $44,6 \%$ restants a été constitués de populations allogènes issues de la sous-région Ouest Africaine (Tableau 4). Les producteurs dans leur majorité adoptaient des pratiques locales de culture du plantain $(99,2 \%)$ (Tableau 5).

\section{Caractéristiques du matériel végétal cultivé et des sols sous culture de bananier plantain}

Le rejet était le matériel végétal le plus utilisé par les producteurs $(99,2 \%)$ (Tableau 6). Ces rejets provenaient généralement des plantations voisines $(94,6 \%)$, hors de la zone d'étude $(3,8 \%)$ ou rarement de centres de recherche $(1,50 \%)$ (Tableau 7). Les paysans obtenaient gratuitement $(95,40 \%)$ ces rejets ou les achetaient à différents coûts : inférieur ou égaux à $300 \mathrm{~F} \mathrm{CFA}(3,8 \%)$, entre 300 et $600 \mathrm{~F}$ CFA $(0,8 \%)$ (Tableau 8$)$.

\section{Modes de culture du bananier plantain}

Les paysans cultivaient le plantain sur les sols sableux $(80,8 \%)$ ou hydromorphes $(19,2 \%)$ (Tableau 9). Sur ces sols, plusieurs lieux où les rejets étaient plantés ont été recensés : les décharges $(36,1 \%)$, autour des maisons $(20,7 \%)$, dans des bas-fonds $(19,5 \%)$ et faiblement dans les jeunes cocoteraies $(1,8 \%)$ (Figure 2). Au total, 08 appellations de bananiers plantains ont pu être identifiées dans les exploitations visitées. Les Cornes ou variétés locales et la variété Agnrin étaient les plus fréquentes avec $74,3 \%$ et $19,9 \%$ respectivement. Les autres appellations de bananiers plantain étaient présentes chacune à moins de 2\% chacune (Figure 3). Les associations de culture avec les bananiers plantains étaient les plus pratiquées $(66,2 \%)$, contrairement à la monoculture du bananier $(33,8 \%)$ (Tableau 10).

Sur la base de la pratique culturale adoptée par les paysans en fonction du lieu de culture, 1'Analyse Factorielle des Correspondances (AFC) a permis de structurer le type d'association selon le lieu où les bananiers sont cultivés. Les axes 1 et 2 formés ressortent $70,6 \%$ et $28,7 \%$ des informations respectivement, soit un total de $99,3 \%$. L'axe 1 d'inertie $70,6 \%$ différentie les paysans cultivant les bananiers en monoculture ou associées aux cultures vivrières de ceux associés aux cultures maraichères ou pérennes. L'axe 2 d'inertie $28,7 \%$ oppose les paysans qui associent les bananiers à des cultures pérennes de ceux, les associant à des cultures maraichères. Les paysans cultivent plus le bananier en monoculture ou associé au vivrier, dans les décharges ou autour des maisons (Type I). Les associations bananiermaraichères sont plus pratiquées dans les basfonds que dans les plaines (Type II). Des associations cocotier-bananiers ont plus été observées avec des cocotiers en production qu'avec les cocotiers jeunes (Type III) (Figure 4).

Trois types de cultures ont été observés en association avec les bananiers plantains. Ce sont les cultures maraichères, les vivrières et une culture pérenne. Parmi ces cultures associées, les plus prépondérantes étaient le piment $(14,3 \%)$, la banane dessert $(13,5 \%)$, l'aubergine $(12,8 \%)$ et le manioc $(10,2 \%)$. Les cultures telles que le melon $(0,4 \%)$, la papaye $(0,4 \%)$ ou la salade $(0,4 \%)$ étaient faiblement associées au bananier (Figure 5).

Plus de la moitié des producteurs (62,3\%), n'utilisaient pas de fertilisants au cours des cycles du bananier plantain. Cependant, 26,9\% d'entre eux faisaient usage de fertilisants organiques, 3,8\% utilisaient des fertilisants minéraux et $6,9 \%$ faisaient usage 
à la fois de fertilisants minéraux et organiques (Figure 6). Les engrais couramment utilisés par les paysans étaient, la fiente de poulets $(21,1 \%)$, l'urée $(8,2 \%)$, les palmes de cocotier $(4,1 \%)$ et le NPK $(3,4 \%)$ (Figure 7$)$. La fiente, est apportée aux bananiers à différentes doses, inférieures à $5 \mathrm{~kg}(16,9 \%)$, entre 5 et $10 \mathrm{~kg}$ $(5,4 \%)$, entre 10 et $15 \mathrm{~kg}(0,8 \%)$ ou supérieures à $15 \mathrm{~kg}(0,8 \%)$ (Tableau 11). Dans les bananeraies visitées, les paysans en majorité ne faisaient pas usage de pesticide $(88,5 \%)$ (Tableau 12) et, $77,7 \%$ des enquêtés ne pratiquaient pas l'irrigation (Tableau 13).

\section{Difficultés au cours de la culture}

Les mortalités des plants au cours du cycle étaient plus dues à la sécheresse $(46,2 \%)$ qu'au vent $(19,7 \%)$ et moins aux attaques de ravageurs et maladies $(12,6 \%)$ (Figure 8 ). La majorité des pertes en bananiers plantains intervenaient aux stades de fructification
(40,8\%), végétatif $(37,7 \%)$ et faiblement au stade de floraison $(10,8 \%)$ (Figure 9).

\section{Devenir de la récolte}

Les bananiers récoltés étaient destinés à la consommation domestique $(53,7 \%)$, soit vendu directement bord champ $(26,4 \%)$ ou sur les marchés locaux $(19,8 \%)$ (Figure 10). En période d'abondance (d'octobre à mars), les régimes de banane connaissaient une variété de prix, ils étaient vendus entre 1000 et $2000 \mathrm{~F}$ CFA $(25,4 \%)$ puis entre 2000 et $3000 \mathrm{~F}$ CFA $(7,7 \%)$ et à moins de $1000 \mathrm{~F}$ CFA $(4,6 \%)$ (Tableau 14). En période de pénurie (d'avril à septembre), les régimes de banane étaient plus vendus entre 2000 et $3000 \mathrm{~F} \mathrm{CFA}(19,2 \%)$ ou entre 1000 et $2000 \mathrm{~F}$ CFA $(11,5 \%)$. Très peu parmi les paysans vendaient les régimes entre 3000 à 4000 F CFA (6,9\%) (Tableau 15).

Tableau 1: Répartition des producteurs de banane plantain selon le genre.

\begin{tabular}{lcccc}
\hline Genre & Effectif & $\begin{array}{c}\text { Intervalles de confiance } \\
\text { autour des fréquences (\%) }\end{array}$ & Chi-2 & p \\
\hline Masculin & 83 & $55,6 \%<63,8<72,1 \%$ & 0,97 & 0,0016 \\
Feminin & 47 & $27,9 \%<36,2<44,4 \%$ & 9,97 & \\
\hline Total. & 130 & 100 & & \\
\hline
\end{tabular}

Tableau 2 : Répartition des producteurs de banane plantain selon les tranches d'âge $(\mathrm{N}=130)$.

\begin{tabular}{lllll}
\hline Tranches d'âge & Effectif & $\begin{array}{c}\text { Intervalles de confiance } \\
\text { autour des fréquences (\%) }\end{array}$ & Chi-2 & p \\
\hline Inférieur ou égale à 20 ans & 3 & $0,0 \%<2,3<4,9 \%$ & & \\
Entre 20-29 ans & 11 & $3,7 \%<8,5<13,2 \%$ & 74,62 & 0,0001 \\
Entre 30 et 39 ans & 22 & $10,5 \%<16,9<23,4 \%$ & \\
Entre 40 et 49 ans & 35 & $19,3 \%<26,9<34,5 \%$ & \\
Supérieur ou égale à 50 ans & 59 & $36,8 \%<45,4<53,9 \%$ & \\
\hline Total & 130 & & 100 & \\
\hline
\end{tabular}


Tableau 3 : Répartition des producteurs de banane plantain selon le niveau d'instruction $(\mathrm{N}=130)$.

\begin{tabular}{lcccc}
\hline Formations & Effectif & $\begin{array}{c}\text { Intervalles de confiance } \\
\text { autour des fréquences }(\%)\end{array}$ & Chi-2 & p \\
\hline Primaire & 27 & $13,8 \%<20,8<27,7 \%$ & & \\
Secondaire & 30 & $15,8 \%<23,1<30,3 \%$ & & \\
Superieur & 3 & $0,0 \%<2,3<4.9 \%$ & 96,46 & \\
Non scolarisé & 65 & $41,4 \%<50,0<58,6 \%$ & & \\
Ecole Coranique & 5 & $0,5 \%<3,8<7,1 \%$ & \\
\hline Total & 130 & 100 & \\
\hline
\end{tabular}

Tableau 4 : Répartition des producteurs de banane plantain selon la nationalité ( $\mathrm{N}=130)$.

\begin{tabular}{lcccc}
\hline Nationalité & Effectif & $\begin{array}{c}\text { Intervalles de confiance } \\
\text { autour des fréquences (\%) }\end{array}$ & Chi2 & P \\
\hline Burkinabé & 32 & $17,2 \%<24,6<32,0 \%$ & & \\
Ivoirienne & 72 & $46,8 \%<55,4<63,9 \%$ & & \\
Nigerienne & 3 & $0,0 \%<2,3<4,9 \%$ & & \\
Togolaise & 4 & $0,1 \%<3,1<6,0 \%$ & 213,86 & 0,0001 \\
Béninoise & 7 & $1,5 \%<5,4<9,3 \%$ & & \\
Ghanéenne & 2 & $0,0 \%<1,5<3,6 \%$ & & \\
Malienne & 10 & $3,1 \%<7,7<12,3 \%$ & & \\
\hline Total & 130 & 100 & & \\
\hline
\end{tabular}

Tableau 5 : Répartition des paysans enquêtés selon l'origine de la formation $(\mathrm{N}=130)$.

\begin{tabular}{|c|c|c|c|c|}
\hline $\begin{array}{l}\text { Information pour la } \\
\text { culture }\end{array}$ & Effectif & $\begin{array}{c}\text { Intervalles de confiance } \\
\text { autour des fréquences }(\%)\end{array}$ & Chi-2 & $\mathbf{p}$ \\
\hline Centre de formation & 1 & $0,0 \%<0,80<2,3 \%$ & \multirow[b]{2}{*}{126,03} & \multirow[b]{2}{*}{0,0001} \\
\hline Pratique locale & 129 & $97,7 \%<99,2<100,7 \%$ & & \\
\hline Total & 130 & 100 & & \\
\hline
\end{tabular}

Tableau 6 : Nature du plant de banane plantain mis en terre par les paysans enquêtés $(\mathrm{N}=130)$.

\begin{tabular}{llccc}
\hline Nature du plant & Effectif & $\begin{array}{c}\text { Intervalles de confiance } \\
\text { autour des fréquences (\%) }\end{array}$ & Chi-2 & p \\
\hline Rejet & 129 & $97,7 \%<99,2<100,7 \%$ & & \\
Vivo plants & 1 & $0,0 \%<0,8<2,3 \%$ & 126,03 & 0,0001 \\
\hline Total & 130 & 100 & & \\
\hline
\end{tabular}


Tableau 7 : Lieu de provenance des rejets à l'origine des bananeraies $(\mathrm{N}=130)$.

\begin{tabular}{lcccc}
\hline $\begin{array}{l}\text { Origine de la } \\
\text { semence }\end{array}$ & Effectif & $\begin{array}{c}\text { Intervalles de confiance } \\
\text { autour des fréquences (\%) }\end{array}$ & Chi-2 & p \\
\hline $\begin{array}{l}\text { Centre de recherche } \\
\text { Plantations }\end{array}$ & 2 & $0,0 \%<1,5<3,6 \%$ & 219,80 & 0,0001 \\
$\begin{array}{l}\text { voisines } \\
\text { Plantations } \\
\text { hors de votre }\end{array}$ & 5 & $0,7 \%<94,6<98,5 \%$ & & \\
zone & & & \\
\hline Total & 130 & 100 & \\
\hline
\end{tabular}

Tableau 8 : Coût des rejets de bananier plantain $(\mathrm{N}=130)$.

\begin{tabular}{lcccc}
\hline Cout du rejet & Effectif & $\begin{array}{c}\text { Intervalles de confiance } \\
\text { autour des fréquences (\%) }\end{array}$ & Chi-2 & p \\
\hline Inf. ou égale à 300 F CFA & 5 & $0,5 \%<3,8<7,1 \%$ & & \\
Entre 300 et 600 F CFA & 1 & $0,0 \%<0,8<2,3 \%$ & 225,43 & 0,0001 \\
gratuit & 124 & $91,8 \%<95,4<99,0 \%$ & & \\
\hline Total & 130 & 100 & & \\
\hline
\end{tabular}

Tableau 9 : Sols de culture des bananiers $(\mathrm{N}=130)$.

\begin{tabular}{lcccc}
\hline Sol & Effectif & $\begin{array}{c}\text { Intervalles de confiance } \\
\text { autour des fréquences (\%) }\end{array}$ & Chi-2 & p \\
\hline Sableux & 105 & $74,0 \%<80,8<87,5 \%$ & & \\
Hydromorphe & 25 & $12,5 \%<19,2<26,0 \%$ & 49,23 & 0,0001 \\
\hline Total & 130 & 100 & & \\
\hline
\end{tabular}

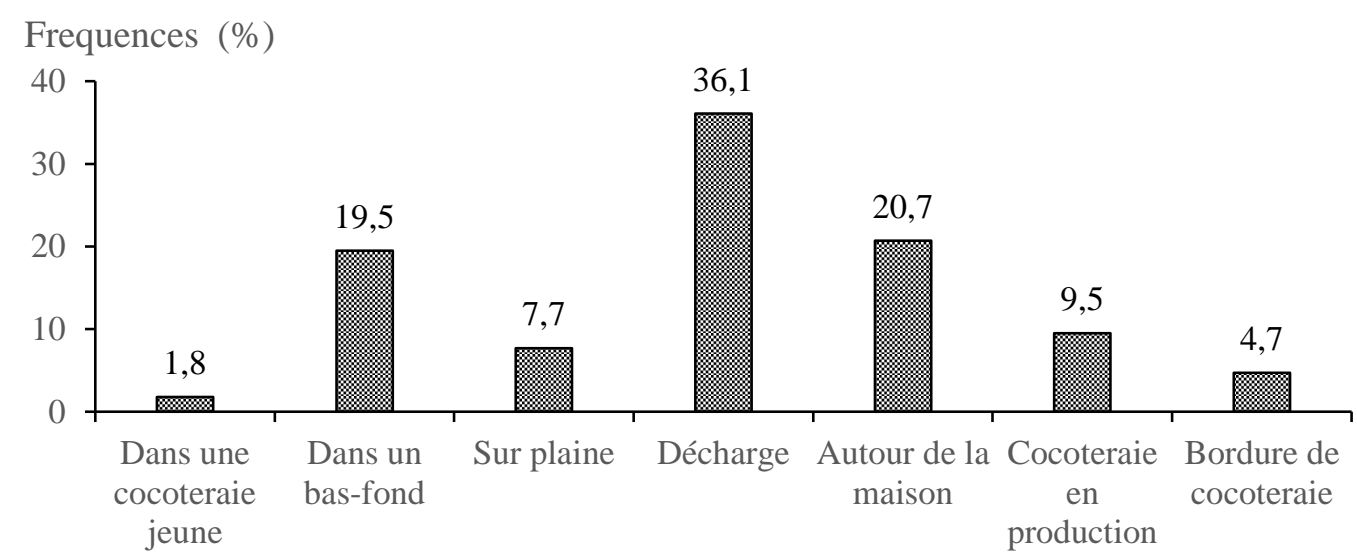

Figure 2 : Lieux de culture adoptés par les paysans enquêtés. 


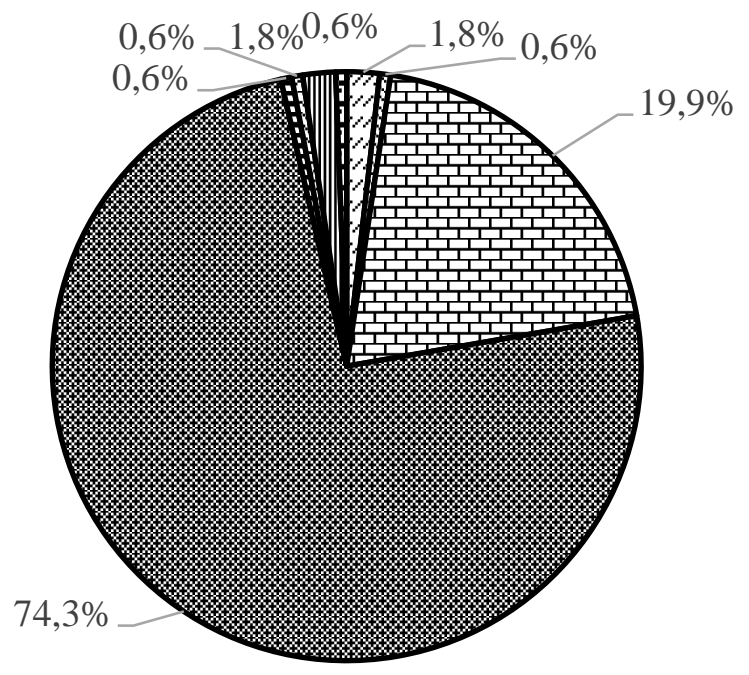
曰PITA 3
DFHIA 21
界旾nrin
Corne
$\boldsymbol{\theta}$ Akotoba
曰Monayo
四Cameroun
曰FHIA 17

Figure 3 : Fréquence des bananiers rencontrés selon leur appellation locale.

Tableau 10 : Pratique culturale adoptée par les paysans $(\mathrm{N}=130)$.

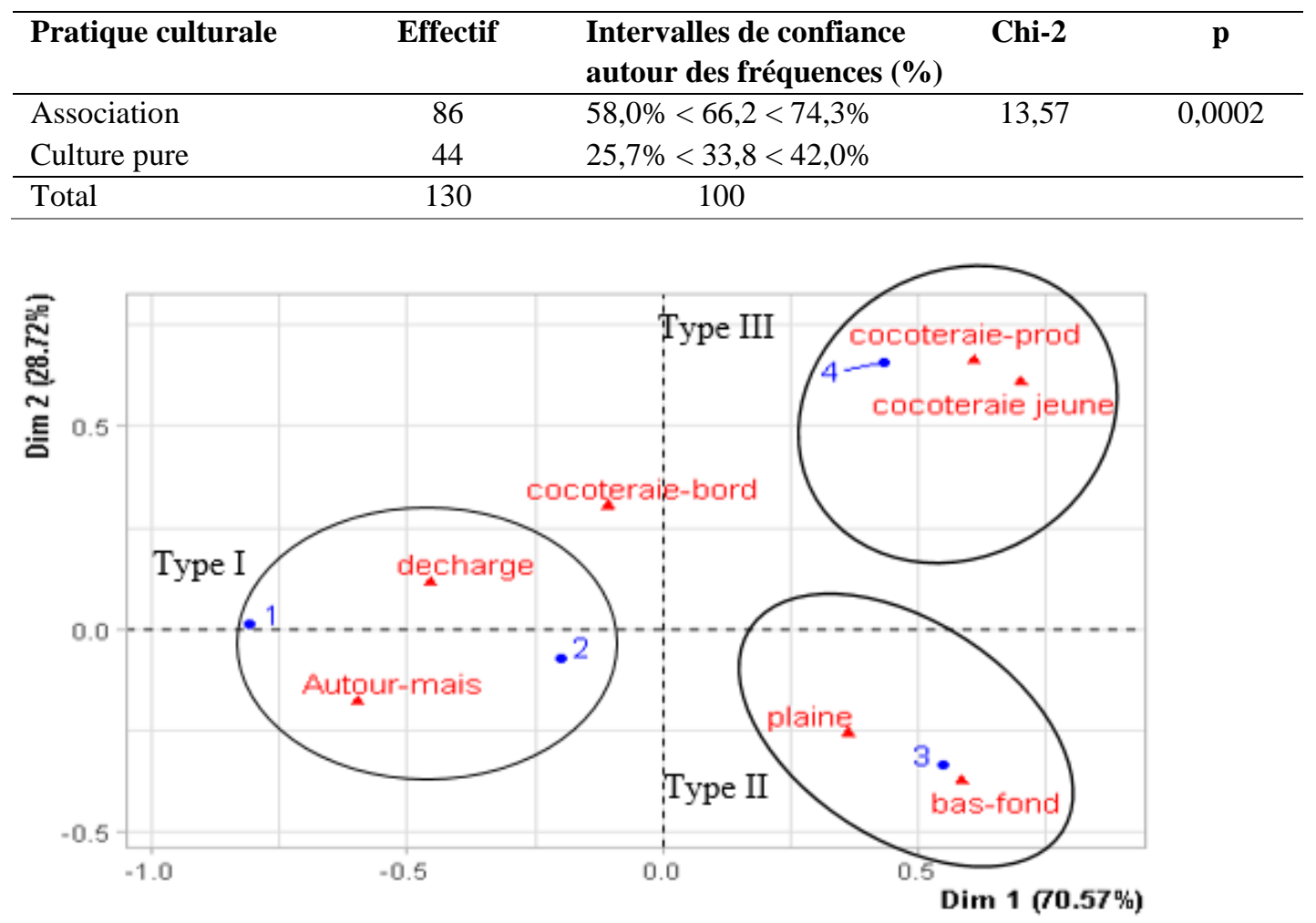

Figure 4 : Analyse factorielle du mode de culture du bananier selon les lieux de culture.

$1:$ bananiers plantains purs $; 2:$ bananiers plantains et vivriers $; 3:$ bananiers plantains et maraichers $; 4:$ bananier plantains et cocotiers ; decharge : décharge ; Autour-mais : autour de la maison ; cocoteraie-bord : bordure de cocoteraie. 


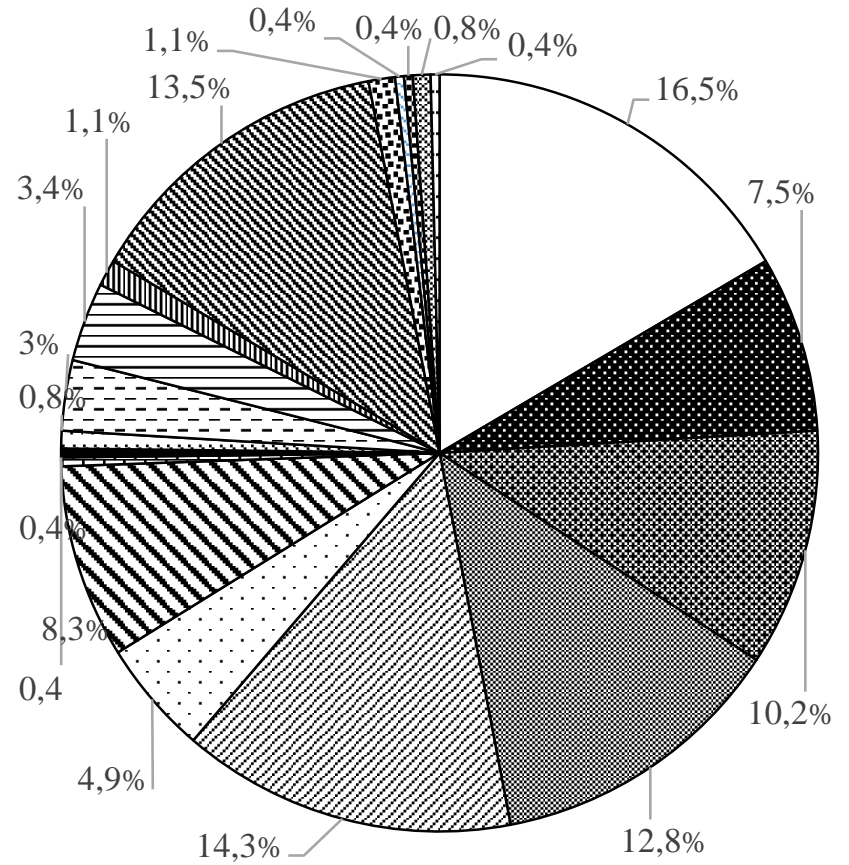

$\square$ pas d'association

因 Manioc

a Piment

$\mathbf{v}$ Gombo

- Choux

$\varpi$ Courgettes

m Taro

Igname

Q Papaye

- Salade
Cocotier

圆 Aubergine

$\square$ Tomate

曰Patate

จais

曰Comcombre

\$ Banane dessert

$\square$ Melon

․․․ Feuilles Daa

Figure 5 : Fréquences des cultures associées aux bananiers plantains.

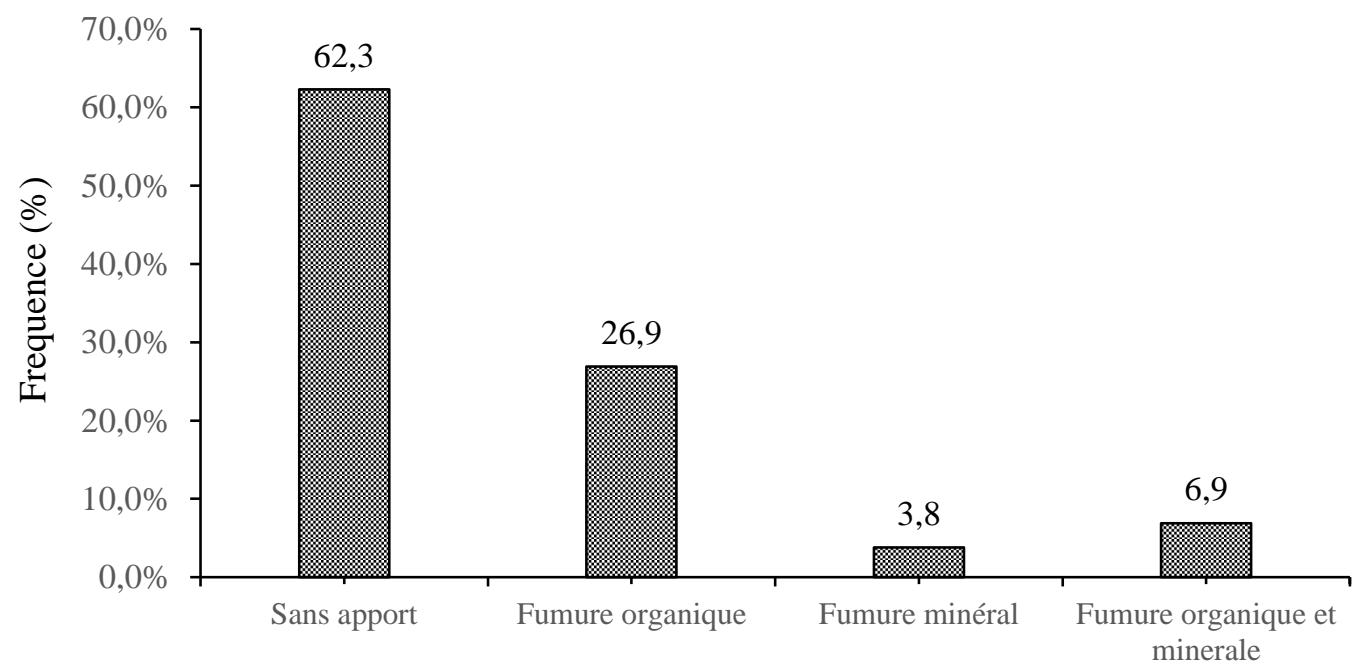

Figure 6 : Traitements des bananiers plantains dans les exploitations. 

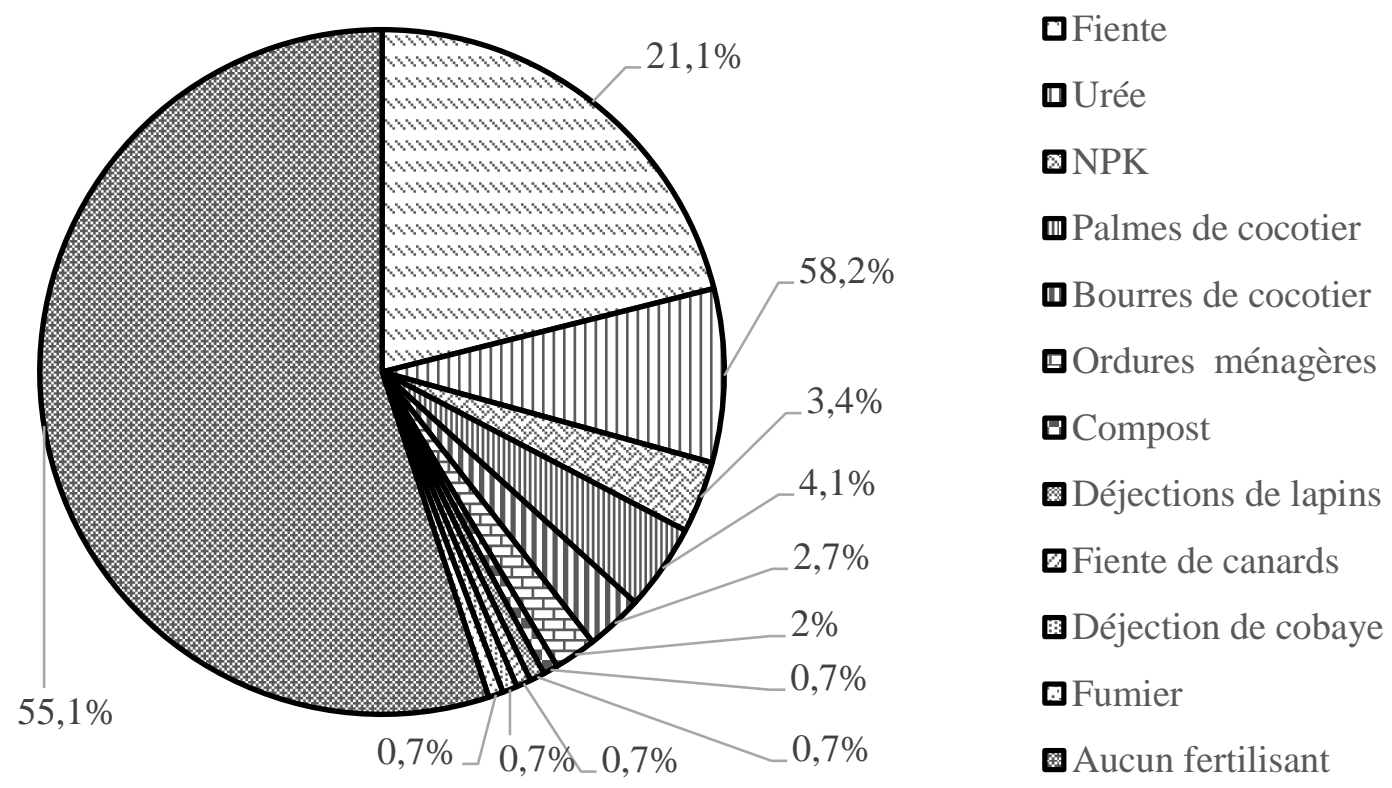

Figure 7 : Fréquences des fertilisants utilisés dans par les producteurs.

Tableau 11 : Quantités de fiente de poulets apportées par pied de bananier $(\mathrm{N}=130)$.

\begin{tabular}{lcccc}
\hline Apports en fiente & Effectif & $\begin{array}{c}\text { Intervalles de confiance } \\
\text { autour des fréquences (\%) }\end{array}$ & Chi-2 & p \\
\hline Inférieur ou égale à $5 \mathrm{~kg}$ & 22 & $10,5 \%<16,9<23,4 \%$ & & \\
Entre $5-10 \mathrm{~kg}$ & 7 & $1,5 \%<5,4<9,3 \%$ & & \\
plus de $15 \mathrm{~kg}$ & 1 & $0,0 \%<0,8<2,3 \%$ & 276,54 & 0,0001 \\
Entre $10-15 \mathrm{~kg}$ & 1 & $0,0 \%<0,8<2,3 \%$ & & \\
Non usage & 99 & $68,8 \%<76,2<83,5 \%$ & & \\
\hline Total & 130 & 100 & \\
\hline
\end{tabular}

Tableau 12 : Fréquences d'utilisations de pesticides sur les bananiers $(\mathrm{N}=130)$.

\begin{tabular}{lcccc}
\hline Pesticide & Effectif & $\begin{array}{c}\text { Intervalles de confiance } \\
\text { autour des fréquences (\%) }\end{array}$ & Chi2 & P \\
\hline Usage & 15 & $6,0 \%<11,5<17,0 \%$ & 76,92 & 0,0001 \\
Non usage & 115 & $83,0 \%<88,5<94,0 \%$ & & \\
\hline Total & 130 & 100 & & \\
\hline
\end{tabular}

Tableau 13 : Frequences d'irrigation des bananiers $(\mathrm{N}=130)$.

\begin{tabular}{lclcc}
\hline Irrigation & Effectif & $\begin{array}{c}\text { Intervalles de confiance } \\
\text { autour des fréquences (\%) }\end{array}$ & Chi2 & P \\
\hline Usage & 29 & $15,2 \%<22,3<29,5 \%$ & 38,88 & 0,0001 \\
Non usage & 101 & $70,5 \%<77,7<84,8 \%$ & & \\
\hline Total & 130 & 100 & & \\
\hline
\end{tabular}




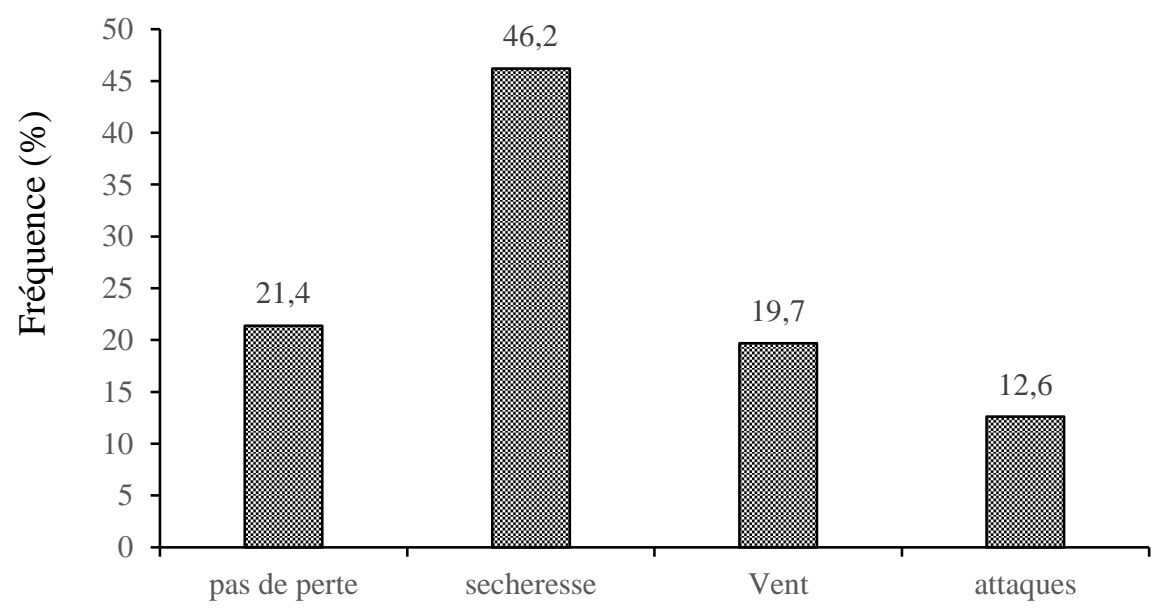

Figure 8 : Fréquences des pertes au cours de la culture.
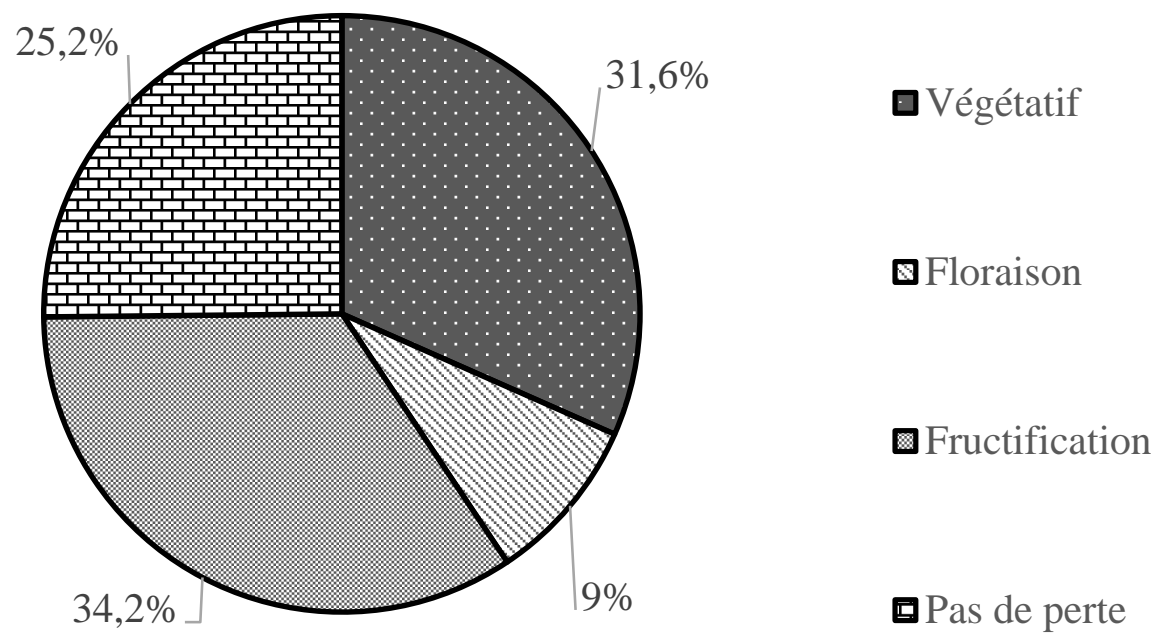

Figure 9 : Fréquences des stades de pertes des bananiers plantains. 


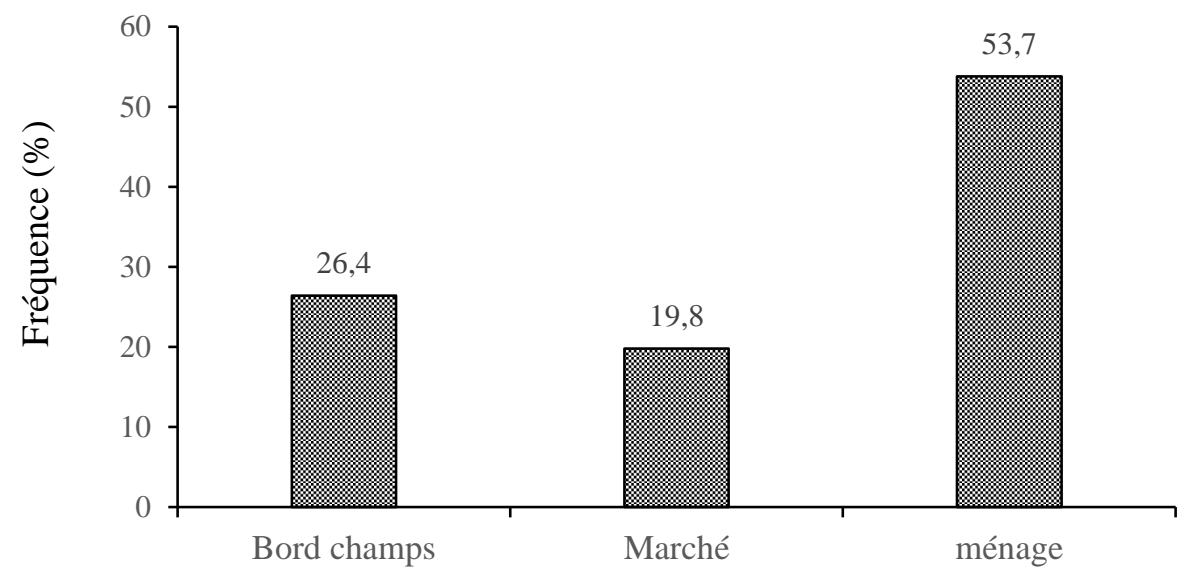

Figure 10 : Devenir de la récolte.

Tableau 14 : Coût du régime en période d'abondance $(\mathrm{N}=130)$.

\begin{tabular}{lcccc}
\hline Coût & $\begin{array}{l}\text { Effecti } \\
\text { f }\end{array}$ & $\begin{array}{l}\text { Intervalles de confiance } \\
\text { autour des fréquences } \\
(\%)\end{array}$ & Chi2 & P \\
\hline Entre 500-1000 FCFA & 6 & $1,0 \%<4,6<8,2 \%$ & & \\
Entre 1000-2000 FCFA & 33 & $17,9 \%<25,4<32,9 \%$ & 109,57 & 0,0001 \\
Entre 2000-3000 FCFA & 10 & $3,1 \%<7,7<12,3 \%$ & & \\
consommation domestique & 81 & $54,0 \%<62,3<70,6 \%$ & & \\
\hline Total. & 130 & 100 & & \\
\hline
\end{tabular}

Tableau 15 : Coût des régimes en période de pénurie $(\mathrm{N}=130)$.

\begin{tabular}{lcccc}
\hline Coût & Effectif & $\begin{array}{c}\text { Intervalles de } \\
\text { confiance autour des } \\
\text { fréquences (\%) }\end{array}$ & Chi 2 & P \\
\hline Entre 1000-2000 FCFA & 15 & $6,0 \%<11,5<17,0 \%$ & & \\
Entre 2000-3000 FCFA & 25 & $12,5 \%<19,2<26,0 \%$ & 100,52 & 0,0001 \\
Entre 3000-4000 FCFA & 9 & $2,6 \%<6,9<11,3 \%$ & & \\
consommation domestique & 81 & $54,0 \%<62,3<70,6 \%$ & \\
\hline Total & 130 & 100 & \\
\hline
\end{tabular}




\section{DISCUSSION}

L'enquête sur l'état des lieux de la culture de la banane plantain à la Station Marc DELORME et dans les villages riverains, sur 130 paysans a révélé que $63,8 \%$ des propriétaires de bananeraie sont des hommes et $32,2 \%$ des femmes. Travaillant sur l'agriculture urbaine et les stratégies de survie des ménages pauvres dans le complexe spatial du district d'Abidjan, Olahan (2010), a indiqué une agriculture essentiellement tenue par les hommes. Ceux-ci travaillent en association, avec leurs femmes, leurs enfants et souvent avec de la main d'œuvre. Le pourcentage élevé d'hommes dans les activités agricoles périurbaines, en zone littorale a également été relevé par Yèhouénou et al. (2009) au sud du Bénin. Par ailleurs, la culture du plantain nécessiterait des efforts musculaires intenses (défrichage, rabattage, tuteurage, récolte des régimes lourds) justifiant la forte implication des hommes. Des résultats similaires sur la dominance des hommes dans la production de la banane ont été également obtenus par Tchuisseu Tchepnkep (2007) au Cameroun et Bakouétila et al. (2016) en République Démocratique du Congo.

La production de la banane était effectuée par des personnes de différentes tranches d'âge. La tranche jeune (jusqu'à 29 ans) était moins active contrairement aux adultes (40-49 ans) et à ceux de plus de 50 ans. Ces jeunes des zones périurbaines seraient donc impliqués dans des activités lucratives autres que l'agriculture ou engagés dans des formations nécessitant leur déplacement vers la ville.

Les résultats de l'enquête ont indiqué une diversité de niveau d'instruction chez les producteurs. Cependant, 50\% n'ont pas suivi une formation académique. $\mathrm{Ce}$ résultat corrobore celui d'Adiko et al. (2010), qui a trouvé que $50 \%$ de la population effectuant l'activité agricole urbaine était non scolarisée. La fréquence de producteurs instruits de notre zone d'étude est certes moyenne, mais comporte très peu de personnes ayant reçu une formation supérieure. Cela serait un inconvénient majeur pour la culture du plantain. En effet, un faible niveau d'instruction des planteurs faciliterait l'intrusion de matériels végétaux peu performants et maintiendrait la culture du plantain à une étape d'agriculture rudimentaire, très peu soucieuses des conditions environnementales. Contrairement, le bon niveau d'instruction constitue un atout important, favorable à l'introduction d'innovations techniques (adoption des nouvelles variétés) et au respect des fiches techniques. Plusieurs études ont montré l'importance d'un niveau d'instruction élevé sur l'adoption des innovations techniques et sur l'efficacité de la production (Tchoumboué et al., 2001 ; Coelli et Fleming, 2004 ; Nkapnang, 2011, Bakouétila et al., 2016).

La population qui cultive la banane dans la zone est composée à 55,4\% d'Ivoiriens. Selon Olahan (2010), la population locale pratiquant l'agriculture en milieu urbain ou périurbain provient essentiellement de l'intérieur du pays. La population allogène enquêtée $(44,6 \%)$, était originaire de la sousrégion avec en tête les Burkinabés. Ce résultat a été corroboré par Olahan (2010) qui a indiqué qu'en milieu urbain (Abidjan), les populations agricoles allogènes provenaient des pays limitrophes et à la base, seraient venus effectuer des travaux champêtres dans des plantations privées ou de l'Etat. Au niveau du littoral ivoirien, la main d'œuvre étrangère constituait l'essentiel du personnel disponible dans les années 1970. Celle-ci s'occupait des plantations de cocotier de l'IRHO (actuel CNRA) et de palmier de la SODEPALM (Amenan et al., 2012).

Les paysans n'étaient pas formés à la culture du bananier $(0,8 \%)$ et adoptaient des pratiques culturales ancestrales ou empiriques $(99,2 \%)$. Cela stipulerait que l'initiation à la culture du bananier aurait été reçue des parents ou d'une connaissance. Selon les réponses recueillies auprès des producteurs, le rejet est le matériel végétal le plus utilisé pour la culture (92,2\%). En effet l'utilisation de vivo plants ou de vitro plants serait peu connue ou très couteux. Par ailleurs ces matériels pourraient être sensibles aux conditions de sols et/ou de climat spécifiques à cette zone sableuse, justifiant leur faible utilisation. 
Les rejets utilisés par les paysans provenaient généralement des plantations voisines L'origine en grande partie locale de ces rejets, mettrait en doute la qualité du matériel végétal utilisé pour la culture et relèverait un risque sanitaire lié à leur utilisation. Le fait d'acquérir gratuitement les rejets $(95,4 \%)$ éveillerait très peu chez les paysans la crainte de d'essuyer de lourdes pertes en culture.

Les bananiers plantains de notre zone d'étude étaient cultivés sur les sols sableux $(80,8 \%)$ et sur les sols hydromorphes $(19,2 \%)$. Sur ces sols, les producteurs plantaient les rejets dans des décharges $(36,1 \%)$, proche de leur lieu d'habitation $(20,7 \%)$, dans des basfonds $(19,5 \%)$ et très peu dans les jeunes cocoteraies $(1,8 \%)$. Les sols sableux du littoral ivoirien en particulier sont très pauvres en éléments minéraux (Lékadou et al., 2009; Kouakou et al., 2019) et sont dotés de grandes capacité de ressuyage accentuant l'assèchement après une pluie. Les paysans auraient de ce fait recours sur ces sols à des endroits très humides, regorgeant de matière minérale et/ou organique à tout moment, dont des décharges, les bas-fonds et autour de la maison. Les décharges sont des lieux de déversement de matières organiques et minérales et sont souvent utilisées par les populations comme lieux de culture de diverses spéculations (Yao, 2014). Cependant, elles demeurent des lieux par excellence d'accumulation des métaux lourds et autres éléments traces (Yao et al., 2014 ; Adjiri et al., 2019). Les bananes consommées par les populations de ces zones pourraient présenter d'énormes risques sanitaires. Par ailleurs, un agriculteur bien formé est un avantage pour la filière, pour les consommateurs, de par la production de denrées de qualité (Sailly et Lebrun, 1997). Pour Adiko et al. (2010), le système agricole en Côte d'Ivoire est déficitaire, car le soin de donner aux populations une formation est typiquement alloué aux autorités sanitaires et agricoles locales. Cette éducation serait donc insuffisante, car convenablement menée, elle pourrait conduire les paysans à la culture du bananier en dehors des lieux à risque comme les décharges et à une utilisation raisonnée des fertilisants.

Des huit variétés identifiées durant l'enquête, la variété CORNE (74,3\%) et la variété Agnrin (19,9\%) étaient les plus rencontrées. Les autres étaient présentes à moins de $2 \%$ chacune. Cette dominance de seulement deux variétés sur huit montre que les paysans n'utilisent que le matériel végétal connu et rependu dans la zone. Aussi, ces bananes sont-elles produites parce qu'elles pourraient présenter des caractéristiques physiques et gustatives bien meilleures que les autres variétés. Comme Bakouétila et al. (2016) au Congo, les résultats de cette enquête ont permis de mettre en évidence l'existence de deux systèmes de cultures du bananier: la monoculture extensive rencontrée à une fréquence de $33,8 \%$ et l'association culturale $(66,2 \%)$. Travaillant sur les systèmes de production du bananier dans l'agriculture urbaine et périurbaine de Yaoundé, Lemeilleur et al. (2003), ont obtenu également deux systèmes de cultures : l'association culturale (bananiers-vivriers, bananiers-ananas, bananiers-palmier à huile) et la monoculture pratiquée par $23 \%$ de producteurs. Des résultats identiques ont été également obtenus en milieu paysan en Côte d'Ivoire, où les bananiers sont presque toujours associés à des cultures industrielles et/ou vivrières (Traoré et al., 2009). De même, les études de Dhed'a et al., (2019), sur la culture du plantain dans les zones agroécologiques en République Démocratique du Congo, ont identifiés ces deux systèmes de culture parmi six rencontrés.

En zone de bas-fond les bananiers sont associés à des cultures maraichères. Selon Yèhouénou et al. (2009), les systèmes de culture maraîchère sur le littoral sont de type intensif avec une utilisation de nombreux intrants sur des surfaces limitées. En effet, dans les sols hydromorphes, en plus de bénéficier de la matière organique décomposée et d'une bonne humidité du sol, les bananiers plantés dans ces zones profiteraient également des engrais apportés aux cultures maraichères. Ce type d'association où le bananier profite des fertilisant apportés aux cultures maraichères permet une gestion durable de l'exploitation, 
mais est aussi pratiqué dans un souci de sécurité alimentaire et de diversification de revenu (Bakouétila et al., 2016).

Les bananiers plantains étaient associés à plusieurs variantes selon qu'ils soient cultivés proches des habitations ou éloignés dans les basfonds. Les plus recensés étaient le piment $(14,3 \%)$, la banane dessert $(13,5 \%)$ l'aubergine $(12,8 \%)$ ou le manioc $(10,2 \%)$. L'adoption de ces spéculations par ces paysans pourrait être due aux conditions de sol et de climat qui faciliteraient leur culture ou au fait que ces denrées feraient partie des aliments les plus consommées ou les plus commercialisées sur les marchés locaux. Les bananes récoltées étaient plus destinées à la consommation domestique $(53,8 \%)$. Certains producteurs procédaient à la vente de leur récolte bord champ $(26,4 \%)$ ou sur les marchés locaux $(19,8 \%)$. Ainsi, les bananeraies rencontrées à la station et ses environs s'inscrivent plus dans un système d'agriculture de subsistance ou le surplus est commercialisé. Cette agriculture est qualifiée d'orpheline selon Mazoyer et Roudart (2002). Le plantain constitue de ce fait, une source de revenu pour les paysans.

Les prix des régimes de bananes connaissent de grandes variations dans les périodes d'abondance et de pénurie définies par le FIRCA, (2014). En période d'abondance (d'octobre à mars), les prix bord champ étaient compris entre 500 et $3000 \mathrm{~F}$ CFA. En période de pénurie (d'avril à septembre), les coûts variaient entre 1000 et $4000 \mathrm{~F} \mathrm{CFA}$.

\section{Conclusion}

Cette enquête sur l'état des lieux de la culture du plantain a été effectuée en zone de cocoteraie, au Sud-Est d'Abidjan, sur le littoral. Elle a eu pour but de faire la typologie des producteurs de plantain, de déceler le matériel végétal le plus utilisé et le mode de culture du plantain. Aussi, a-t-elle permis de connaitre les contraintes liées à cette culture et de savoir le devenir des récoltes. Les résultats montrent que les producteurs de banane sont en majorité des hommes. La tranche d'âge de producteur la plus active est celle d'au moins 50 ans. Le rejet est le matériel végétal le plus utilisé pour la culture. Le bananier plantain est cultivé soit en monoculture ou associées à plusieurs variantes. Cependant, très peu d'association cocotier-bananier ont été observés. Nombreux ont été les producteurs ne procédant pas à la fertilisation des bananier plantains, à leur irrigation ou à leur entretien phytosanitaire. Les mortalités des plants au cours du cycle étaient plus dues à la sécheresse qu'au vent et moins aux attaques de ravageurs et maladies. Les fruits récoltés étaient plus consommés dans les ménages que vendus bord champ et sur les marchés locaux. Les plantains produits en zone littorale sont d'un grand intérêt socioéconomique pour les populations. Ainsi, il serait important d'envisager à grande échelle cette culture dans les interlignes de cocotier, toutefois avec un itinéraire technique bien défini et maitrisé. Cette technique culturale permettrait un revenu substantiel au nuciculteur en attendant l'entrée en production des cocotiers.

\section{REFERENCES}

Adjiri O, Any-Grah A, Kouamé K, Kouassi E, Biémi J. 2019. Risques toxicologiques de la cohabitation avec une décharge : cas d'Akouédo en Côte d'Ivoire. Santé Publique, 31(4): 567-579. DOI : 10.3917/spub.194.0567.

Amenan Y, Atcham A, Pohe J, D'Almeida MA, Zama P. 2012. Association d'organismes de type mycoplasmes avec le dépérissement mortel des cocotiers de Grand-Lahou en Côte d'Ivoire. International Journal of Biological and Chemical Sciences, 6(3): 959 - 984. DOI: 10.4314/ijbcs.v6i3.5.

Assa R. 2007. Diagnostic de la cocoteraie du littoral ivoirien : étude physico-chimique, microbiologique et organoleptique de l'eau et de l'amande des fruits de quatre cultivars du cocotier (Cocos nucifera $\mathrm{L}$.) selon les stades de maturité. Thèse de doctorat unique, Université de Cocody, Abidjan, Côte d'Ivoire, 188 p.

Brou T. 2005. Climat, mutations socioéconomique et paysages en Côte d'Ivoire. Rapport d'activités scientifique, pédagogique, administrative et publications en appui au mémoire de 
synthèse. Université des Sciences et Technologies de Lille, 106 p.

CNRA. 2005. Bien cultiver la banane plantain en Côte d'Ivoire. Centre National de Recherche Agronomique, Fiche technique, $4 \mathrm{p}$.

Coelli TJ, Fleming E. 2004. Diversification economies and specialization efficiencies in a mixed food and coffee smallholder farming system in Papua New Guinea. Agricultural Economics, 3(1): 229 - 239. https://doi.org/10.1111/j.15740862.2004.tb00260.x.

Dhed'a D, Adheka GJ, Onautshu O, Swennen R. 2019. La Culture des Bananiers et Plantains dans les Zones Agroécologiques de la République Démocratique $d u$ Congo. Presse Universitaire UNIKIS : Kisangani ; 72p.

Dhed'a DB, Moango MA, Swennen R. 2011. La culture des bananiers et bananiers plantains en République Démocratique du Congo. Support didactique, Saint Paul, Kinshasa, 85 p.

Diabagaté S. 2008. Le littoral ivoirien face aux risques naturels. Rapport de séminaire de DEA. Université de Cocody, Abidjan, 23 p.

FIRCA 2014. Les filières fruitières. Bulletin d'information du fonds interprofessionnel pour la recherche et conseil agricole, 48 p.

Koffi Z. 2016. Caractérisations agromorphologique et moléculaire de quelques descendances hybrides de cocotier (Cocos nucifera L.) NJM x GVT en Côte d'Ivoire et évaluation de la tolérance à Pseudotheraptus devastans Distant (Hetero0ptera: Coreidae). Thèse de Doctorat de l'Université Nangui Abrogua, Abidjan, Côte d'Ivoire, 145 p.

Kouakou K, Yao K, Sika A, Gogbeu S, Koné L, Dogbo D. 2019. Caractérisation de l'activité de maraîchage dans la commune de Port-Bouët (Abidjan, Côte d'Ivoire). Journal of Animal \& Plant Sciences, 41(1): $\quad$ 6747-6756. DOI: https://doi.org/10.35759/JAnmPlSci.v411.2 .
Kouame N, Dick A, Assidjo N, Anno A. 2014. Étude de la croissance du bananier plantain (Musa sp., AAB cultivar Corne 1) dans les régions de Yamoussoukro et Azaguié, Cote D'Ivoire. Journal of Applied Biosciences, 76: 6411-6424.

Kwa M, Temple L. 2019. Le Bananier Plantain: Enjeux Socio-économiques et Techniques. Quæ CTA Presses Agronomiques de Gembloux ; 199 p.

Lassois L, Busogoro JP, Jijakli H. 2009. La banane : de son origine à sa commercialisation. Biotechnology, Agronomy, Society and Environment, 13(4): 575 - 586.

Lékadou TT, N'guessan A, Konan JL, Zakra N, Yao KA, Allou K. 2009. Effet de la densité sur les paramètres dendrométriques d'Acacia auriculiformis en association avec le cocotier (Cocos nucifera L.) sur sables quaternaires en Côte d'Ivoire. Agronomie Africaine, 21(1) : $71 \quad-\quad 82 . \quad$ DOI: 10.4314/aga.v21i1.46210.

Lemeilleur S, Temple L, Kwa M. 2003. Identification des systèmes de production du bananier dans l'agriculture urbaine et périurbaine de Yaoundé. InfoMusa, 12(1): 13-16.

Loeillet D. 2007. Bananes : [2007]. In : Cyclope 2007 : les marchés mondiaux. Paris: Economica, 334 - 338.

Mazoyer M, Roudart L. 2002. Histoire des agricultures du monde. ULB Institutional Repository 2013/44785, ULB-Université Libre de Bruxelles. https://ideas.repec.org/ulb/ulbeco/201344785.html.

Nkapnang DI. 2011. Identification et optimisation des conditions d'appropriation des innovations sur bananiers et plantains par les producteurs au Cameroun. Thèse de doctorat, Faculté d'ingénierie biologique, agronomique et environnementale, Université Catholique de Louvain, 142 p.

Olahan A. 2010. Agriculture urbaine et stratégies de survie des ménages pauvres dans le complexe spatial du district d'Abidjan. Journal Opening Edition, 
10(2):

$27 \mathrm{p}$.

DOI:

https://doi.org/10.4000/vertigo.10005.

Sailly JC, Lebrun T. 1997. Dix ans d'avancées en économie de la santé, Actes des XIX ${ }^{\circ}$ Journées des Economistes de la Santé. Français, Paris, John Libbey Eurotext, $304 \mathrm{p}$.

Sharrock S, Lusty C. 2000. Nutritive value of banana. In INIBAP annual report 1999 INIBAP: Montpellier, France: 28-31.

Tchoumboué J, Tchouamo IR, Pinta JY, Njia N. 2001. Caractéristiques socioéconomiques et techniques de l'apiculture dans les hautes terres de l'Ouest Cameroun. Tropicultura, 19 (3): $141-146$.

Tchuisseu Tchepnkep RA. 2007. Analyse de la principale filière d'approvisionnement de la ville de Yaoundé en banane plantain. Mémoire de fin d'études d'ingénieur agronome, option économie et sociologie rurales, Faculté d'Agronomie et des Sciences Agricoles, Université de Dschang, $45 \mathrm{p}$.

Thiémélé DEF, Traoré S, Aby N, Gnonhouri P, Yao N, Kobenan K, Konan E, Adiko A,
Zakra N. 2017. Diversité et sélection participative de variétés locales productives de banane plantain de Côte d'Ivoire. Journal of Applied Biosciences, 114: 11324-11335.

Traoré S, Kobenan K, Kouassi KS, Gnonhouri G. 2009. Systèmes de culture du bananier plantain et méthodes de lutte contre les parasites et ravageurs en milieu paysan en Côte d'Ivoire. Journal of Applied Biosciences, 19 : 1094 - 1101.

Yao K. 2014. Contamination des cultures maraichères produites sur la décharge d'Akouédo et risques sanitaires associés. Mémoire de Master 2 en Sciences et Gestion de l'Environnement de l'Université Nangui-Abrogua, Abidjan, Côte d'Ivoire, $68 \mathrm{p}$.

Yèhouénou E, Pazou A, Toko AL, Houndantod JZ. 2009. Variabilité des éléments chimiques du sol sableux du littoral amendé avec des sous-produits d'épuration des eaux usées à Sèmè-Podji au Sud-Bénin. Buletin de la Recherche Agronomique du Benin, 64: 27-33. 